\title{
Die Europäische Metropolregion Zürich - die Entstehung des subpolitischen Raumes
}

\section{Heiko Behrendt, Christian Kruse, Zürich}

\section{Von Europäischen Metropolregionen als neue politische Systemebene}

Vom systemischen Ansatz aus betrachtet, sind es Netzwerke strukturierter und raumrelevanter Akteursbzw. Handlungssysteme (bspw. Finanzmarkt, Verkehr, Kommunikation, Arbeit), welche die Stellung von Wirtschafts- und Lebensräumen definieren und die Verbindungen zu anderen Metropolen und Metropolregionen herstellen. Dabei handelt es sich um verschiedene Aktivitätsebenen, die teilweise vertikal und teilweise horizontal miteinander verknüpft sind. Die darin verborgenen Strukturen, die durch z.B. Manager bzw. Arbeitnehmer und Bewohner personifizierbar, durch Unternehmen und Institutionen systemisch beschreib- sowie verortbar werden, bilden ein komplexes System von Kommunikationen und Handlungen. Sie sind die Basis der meist schwer fassbaren metropolitanen Räume - seien sie mono- oder polyzentrisch.

Das Interessante in der Auseinandersetzung mit Metropolregionen liegt in der Verbindung zweier Aspekte: einerseits in der Identifikation funktionaler Verflechtungen, deren Interaktionen losgelöst von politischadministrativen Grenzen wirken, andererseits in der Betrachtung der dadurch ausgelösten Eingriffe in existente politische und sozioökonomische Systemstrukturen, da diese «neuen» räumlichen Verflechtungen nicht mehr mit den tradierten Steuerungs- und Regulationsinstrumenten ausreichend gelenkt werden können. Das Aussergewöhliche hierbei ist, dass zum bestehenden (föderalen) politischen System eine weitere Ebene eines unkonkreten, (noch) nicht institutionalisierten ökonomisch-politischen Systems auf der Ebene von (europäischen) Metropolregionen hinzugefügt wird. Es operiert nach anderen Regeln, Werten und Zielen und agiert durch Akteure, die demokratisch nicht legitimiert sind (vgl. BECK 1986, Habermas 1998, Cerny 1998, Kruse 1999).

\section{2 «Politics without policies» in metropolitanen Systemen}

Die Europäische Union formiert sich und organisiert ihre (informelle, räumliche) Strukturpolitik auf der Basis eines Netzwerkes Europäischer Metropolregionen. Im Kern geht es um die Vernetzung von europäischen Teilräumen, welche die Europäische Union in ihrer Kohärenz stärken soll (EUROPäISCHE Kommission 1999). Metropolregionen sind die treibenden Faktoren im europäischen Einigungsprozess. Der Umgang mit metropolitanen Knotenpunkten ist damit zum Symbol des Überganges von der ökonomischen zur politischen Union geworden, da auf dieser Ebene die zum Subsidiaritätsprinzip verpflichtete Union am wenigsten mit den politisch etablierten Systemen in Konfrontation gerät. Hochgeschwindigkeitsverbindungen, Ausbau des Strassennetzes sowie Intensivierung des innereuropäischen Flugverkehrs schaffen eine interregionale Mobilität, die wesentlich zur europäischen Integration beitragen und dem «Projekt Europa» zum Erfolg verhelfen soll. Die nationalen und lokalen Politiken reagieren auf diese Entwicklungen. Um Wettbewerbsfähigkeit und politische Integration zu gewährleisten, verlassen politische Netzwerke die Ebene administrativ geltender nationaler Gebietskörperschaften und beginnen intensive Kooperationen innerhalb und zwischen den Metropolregionen. Neue Formen der intraregionalen Zusammenarbeit sowie politischer Strukturen für die Steuerung funktionaler Regionen sind in Ansätzen zu erkennen. Der Bericht der OECD über «metropolitan governance» weist verstärkt darauf hin, dass in den nächsten Jahren diese Thematik zunehmend in den Mittelpunkt des politikwissenschaftlichen Demokratiediskurses gelangen wird (OECD 2000).

Doch in der diskurstheoretischen Analyse taucht schnell eine Erkenntnis auf, die in der fachlichen Auseinandersetzung zum Kern innovativer, demokratietheoretischer Lösungen werden wird. Die im föderalen Aufbau der Europäischen Union formal definierten, politischen Entscheidungsstrukturen (vgl. GoodmaN 1998) sowie die «alten» nationalstaatlichen Systemstrukturen passen mit den nicht politisch-administrativ gefassten, polystrukturellen, funktionalen Metropolregionen, von denen zudem die bedeutenden ökonomischen und politischen Interaktionen ausgehen, nicht zusammen. Die in den Metropolregionen existenten Akteurssysteme sind letztlich die Korrespondenzadresse der europäischen Politik. Somit ist ein Zielkonflikt entstanden, der politisch jedoch nicht so einfach zu lösen ist. Formal gesehen, existiert dieses Politikdefizit nicht, da es keine Institution gibt, die dafür zuständig ist. Keine der verschiedenen politischen Ebenen besitzt den Auftrag oder die Kompetenz, neue, innovative Strukturen zu schaffen. In diesem Kontext herrscht Handlungsunfähigkeit. In Realität stehen die 
europäischen Staaten immer deutlicher einer Neugliederung demokratischer Politikstrukturen gegenüber: erstens dem Aufbau bisher noch nicht im Detail durchdachter supranationaler Demokratieformen, zweitens einem Abbau nationalstaatlicher Kompetenzen und drittens der Entstehung eines metropolitanen Systems von «politics without policies». Zahlreiche Initiativen kooperativer Form zwischen Politik und Wirtschaft haben sich zum Sprachrohr der metropolitanen Politik herausgebildet und die subpolitische Vertretung regionaler Anforderungen vor allem im Wettbewerb gegenüber anderen Metropolregionen übernommen. Jedoch ohne direkten demokratischen Auftrag. Ihre Arbeit trägt aufgrund des medialen Interesses im Wesentlichen zur allmählich sich bildenden regionalen Identität eines nach aussen hin wirkenden einheitlichen Wirtschaftsraumes mit hohem Entwicklungspotenzial und von globaler Bedeutung bei.

\section{Europäische Metropolregion Zürich (EMRZ)}

\subsection{Neue Systemstrukturen durch eine Europäische Metropolregion Zürich in der Schweiz?}

Was bedeutet das für die Schweiz? Wenn Europäische Metropolregionen identifizierbar sind und diese (vgl. European Metropolitan Regions Project 1999) immer mehr die Rolle nationaler Motoren und supranationaler Integrationsfunktionen erhalten sowie zunehmend auch eine internationale Vernetzung durch physische Infrastrukturen und wirtschaftlichen Kooperationsleistungen entsteht, dann stellt sich schnell die Frage nach einem komplementären Raum innerhalb der Schweiz.

Ist Zürich eine Europäische Metropolregion, die einerseits durch ihre funktionale Struktur eine vernetzte Wirtschaftsregion ist und andererseits jene wirtschaftliche und politische Integration für die Schweiz trotz Nichtmitgliedschaft innerhalb der Europäischen Union schon leistet? Existieren zudem die oben beschriebenen Prozesse einer Subpolitik auf der Ebene einer nicht institutionalisierten Region? Wird der bisher als europäisches Vorzeigemodell existierende Schweizer Föderalismus und die direkte Demokratie mit dem Aufeinanderprallen zweier Politiksysteme konfrontiert und erhält dadurch auch der politische Diskurs in der Schweiz eine neue Prägung?

Die Schweiz tut sich schwer, eine grossräumige Betrachtung anzugehen. Die Erarbeitung von sieben statistischen Grossregionen zur Kompatibilität mit den Datenformaten der Europäischen Union (vgl. BunDesamt für Statistik 1999, Thierstein et al. 2000) hat noch keine befriedigende Lösung angeboten. Sie entspricht nur in geringen Teilen den Realitäten funktionaler, räumlicher Verflechtungen. Auf wissenschaftlicher Ebene fehlen die Auseinandersetzung und
Überprüfung von Raumkonzepten urbaner Systeme sowie detaillierte Analysen der als einzige in Frage kommenden potenziellen Europäischen Metropolregionen Zürich und Genf-Lausanne. Eine wissenschaftliche Diskussion um Zürich als funktionale Metropolregion innerhalb Europas hat bisher kaum stattgefunden. Das Dilemma steckt vor allem darin, dass bisher noch keine überzeugende Ansätze gemacht worden sind, einen räumlichen Interaktionsperimeter für die Region Zürich zu erstellen. Die Unsicherheit im Umgang mit Begriffen wie Agglomeration, Metropole, Region und gar Stadt ist auf politischer sowie auf wissenschaftlicher Ebene deutlich zu spüren. Das Forschungs- und Diskursdefizit um urbane Systeme in der Schweiz kommt darin zum Ausdruck. Die wenigen vorliegenden Studien zu Zürich argumentieren zwar, dass der Wirtschaftsraum Zürich einen grossen Teil der deutschschweizerischen Kantone umfasse und sogar in den süddeutschen Raum übergehe. Andererseits begrenzen sie ihre Aussagen auf den viel zu kleinen Bezugsraum des Kantons Zürich, da hier vor allem statistische Daten vorliegen. Trotzdem ist auf politischer Ebene intensiv versucht worden, Zürich unter dem Begriff Metropole in ein europäisches Regionenranking einzuordnen. Die Studie der Basler Konjunkturforschung (BAK) zum internationalen Benchmarkreport (ZürCHER KantonalbanK 2000) beschreibt Schlüsselbranchen der Region und bewertet die Standortattraktivität. Zürich wird dabei in den Kreis der europäischen Metropolen eingeordnet. Dennoch ist die Studie unbefriedigend. Sie besitzt erhebliche räumliche Abgrenzungsprobleme und eine ungenaue statistische Erhebung sowie nur mit Einschränkung vergleichbare Referenzdaten anderer europäischer Regionen, die nur grobe Trends, jedoch keineswegs eine genaue Situationsbeschreibung zulassen. Ungeachtet des Nutzens solcher Analysen werden diese Ergebnisse lediglich auf politischer Ebene benötigt, um in politischen Parolen mit Positionsaussagen zur Stellung Zürichs politischen Zielen Nachdruck zu verleihen (vgl. zum Sinn des Unsinns von Rankings den Beitrag von Dangschat 2001).

In internationalen wissenschaftlichen Städterankings nimmt Zürich vor allem wegen seines Finanzplatzes einen Rang als Europäische Metropolregion ein (Reclus 1989, Brugger \& Kärcher 1992, KrätKe 1992, Rossi \& Steiger 1995, Zürcher Kantonalbank 2000). Sie konkurriert trotz ihrer geringen Grösse mit Zentren wie Frankfurt, Hamburg, München, Mailand oder auch Brüssel sowie der Randstad. Und auch die neueste Untersuchung von TAYLOR und WALKER, die auf einer neuen multivariaten Analyse beruht, bescheinigt Zürich den Status einer «Beta world city» auf der gleichen Stufe mit San Francisco, Sydney und Toronto (TAYlor \& WALKer 2001). Jedoch ist die Stellung Zürichs kaum in den internationalen Diskurs um 
Metropolenfunktionen in einem vernetzten Europa eingegliedert worden. Zürich wird weder in der wissenschaftlichen noch in der politischen "community" oder gar auf der Ebene von Wirtschaftsanalysten in Europa wahrgenommen. Einzig die Arbeit von Hitz et al. (1995) hat die Entwicklung Zürichs zur Weltmetropole beschrieben. Sie dokumentieren in diesem Zusammenhang die mit der Metropolenfunktion verbundene «head-quarter-economy» in der Region Zürich. Doch eine tiefgreifende Analyse der Struktur und der Funktionen der Metropole und des treibenden Faktors «Finanzplatz» ist bisher nicht erfolgt. Eines ist dabei deutlich: die Stadt Zürich ist mit seinen 354'000 Einwohnern viel zu klein, um eigenständig metropolitane Funktionen zu tragen. Auch die Erweiterung einer Analyse auf die Agglomeration oder auf den Kanton Zürich reicht nicht aus. Es ist notwendig, im internationalen Diskurs verwendete Ansätze über polyzentrisch strukturierte Metropolregionen in die Analyse eines grossräumigen Wirtschaftsraumes Zürich einzubringen und hierüber quantitative sowie qualitative Daten zu erarbeiten, ähnlich wie dies beispielsweise in der Region Frankfurt/Rhein-Main, der Region Rhein-Ruhr oder der Randstad der Fall ist. Aus diesem Grund ist es ein Anliegen dieses Beitrags, den längst fälligen raumwissenschaftlichen Diskurs mit dem immer stärker auftretenden Phänomen einer polyzentrischen Europäischen Metropolregion Zürich anzugehen.

\subsection{Das Wachsen einer Wahrnehmungsregion Zürich}

Die Entwicklungen, die derzeit in der öffentlichen Debatte um den Wirtschaftsraum Zürich zu beobachten sind, entsprechen einem für polyzentrische Europäische Metropolregionen typischen Prozess. Zunächst standen wirtschaftliche Interessen im Vordergrund, die es notwendig erscheinen liessen, das Label «Standort Zürich» weit über die Grenzen der Stadt und des Kantons Zürich hinaus zu verwenden (vgl. KruSE 2000). Doch ist dies kein institutionell getragener bzw. koordinierter Prozess. Erst mit der 1999 gegründeten Standortmarketing-Organisation Greater Zurich Area - The Zurich Network (GZA) ist in Form einer Kooperation von verschiedenen Kantonen und Städten sowie Vertretern führender Schweizer Unternehmen der Begriff des «Standortes Zürich» quasi institutionalisiert worden. Durch einen sogenannten «One-Stop-Shop» übernimmt die privatwirtschaftlich organisierte GZA eine Schnittstellenfunktion zwischen ansiedelungswilligen Unternehmen und den jeweiligen kantonalen Wirtschaftsförderungen und Verwaltungseinheiten ein. Für die GZA ist der endgültige Standort eines neuen Unternehmens in der Region nicht so bedeutend, solange er im Aktionsgebiet der GZA liegt. Dieses wurde mit zunächst 60 , später dann 90 Minuten Autofahrtzeit vom Flughafen Zürich aus in alle Richtungen definiert. Somit ist ein
Raum von Basel-Flughafen bis Bregenz sowie von Süddeutschland bis an die Grenzen des Tessins und Berns entstanden. Dieser Anspruch ist nicht überall mit Begeisterung aufgenommen worden. Hartnäckige Widerstände in den kantonalen Parlamenten gegen eine Mitgliedschaft bei der in Zürich ansässigen GZAInitiative zeigen das deutlich. Die dargestellten politischen Prozesse in der Regionenbildung sind somit auch in der Schweiz identifizierbar. Im Falle Zürichs ist das Entstehen eines subpolitischen Raumes nach «Vorbild» der Metropolregionen Europas zu beobachten. Mit dieser Entwicklung hat ein schleichendes Umdenken eingesetzt. Es überträgt sich nach und nach von Wirtschaftsakteuren auf Politiker und zunehmend auch auf die Bevölkerung. Die Region Zürich wird unter anderem auch durch die Medien zunehmend als zusammenhängender Wirtschafts- und Lebensraum wahrgenommen.

\section{Metafunktionen Europäischer Metropolregionen}

Die Forschung hat sich in den letzten Jahren daher verstärkt mit funktionalen Strukturen von monound polyzentrischen Metropolregionen auseinandergesetzt. Städte und Regionen mit einem hohen Internationalisierungsgrad werden dabei als bedeutende Knotenpunkte innerhalb des Weltwirtschaftssystems charakterisiert (vgl. z.B. SASSEN 1991, SASSEN 1994, Andersson \& Andersson 2000, Marcuse \& Kempen 2000, Soja 2000, Scotr 2001). Der Diskurs setzt sich einerseits mit strukturellen Fragen, andererseits mit institutionellen Konsequenzen dieser Raumphänomene auseinander (vgl. z.B. BLotevogel 1998a,b, DieLemann \& Faludi 1998, Dear 2000, Soja 2000, Urban StUdies 2001). In der deutschsprachigen Literatur ist verstärkt der Begriff der Europäischen Metropolregion verwendet worden, der eine neue Raumkategorie mit bestimmten Kriterien bezeichnen soll. In Deutschland hat das zur Erkenntnis geführt, dass auf der Ebene der Raumordnungspolitik des Bundes sowie der Bundesländer eine neue raumordnungspolitische Kategorie eingeführt werden muss, um diese Metropolregionen nun auch raumordnungspolitisch besser einordnen zu können. Mit dem Konzept Europäische Metropolregion hat die deutsche Ministerkonferenz für Raumordnung (MKRO) eine neue Ebene in das Zentrale Orte-Prinzip eingefügt, deren räumliche Reichweite die Integrations- und Wettbewerbsfähigkeit des Nationalstaates innerhalb Europas beeinflusst und den Motor der gesellschaftlichen, wirtschaftlichen, sozialen und kulturellen Entwicklung erfassen soll (vgl. BUNDESMINISTERIUM FÜR RAUMORDNUNG, BAUWESEN UND Städtebau 1995, Blotevogel 1998a,b, Michel 1998, Motzkus 2000, RitTer 1997, zur Theorie und Entstehung des Begriffs s. Beitrag von BLotevogel in diesem Band). 


\begin{tabular}{|c|c|}
\hline Funktional-qualitative Merkmale & Systemisch-strukturelle Merkmale \\
\hline 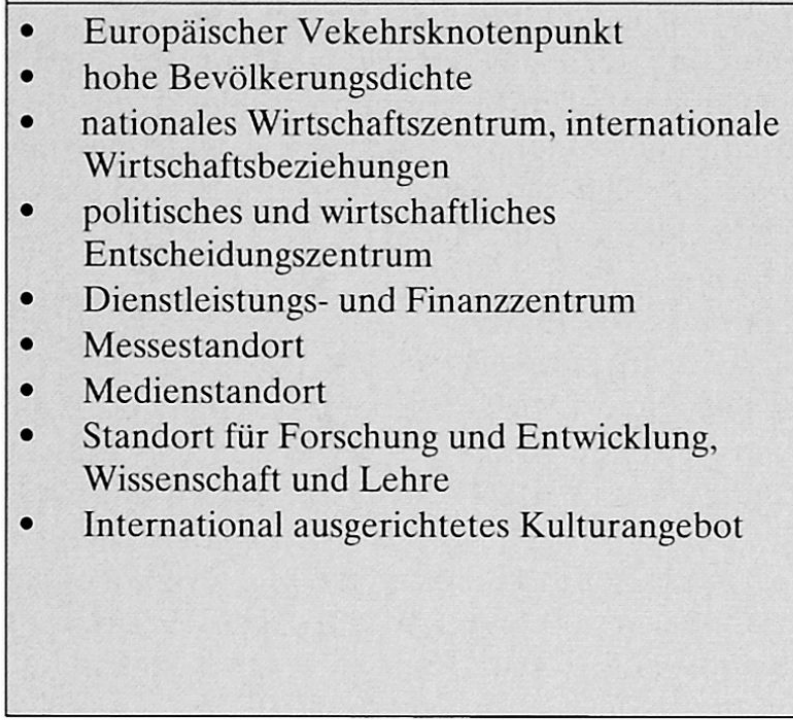 & $\begin{array}{l}\text { - } \text { regionale Verflechtung in einem mono/- } \\
\text { polyzentrischen System des } \\
\text { Metropolregionenkerns und der } \\
\text { Metropolregionenbasis } \\
\text { - } \text { politisch nicht organisiert und nicht } \\
\text { institutionalisiert } \\
\text { subpolitische Regulation durch ein politisch } \\
\text { nicht institutionalisiertes Akteurssystem } \\
\text { Entstehung kooperativer } \\
\text { Zusammenarbeitsformen zwischen Wirtschaft } \\
\text { und Politik in Form privatwirtschaftlich } \\
\text { strukturierter Initiativverbünde, die } \\
\text { metropolitane Politik betreiben } \\
\text { - } \text { "Interfacefunktion» zwischen regionalen, } \\
\text { nationalen und internationalen ökonomischen } \\
\text { und politischen Netzwerken }\end{array}$ \\
\hline
\end{tabular}

Abb. 1: Merkmale Europäischer Metropolregionen Characteristics of European metropolitan regions Caractéristiques des régions métropolitaines européennes Quelle: Blotevogel 1998; eigene Darstellung

Das lenkt die Aufmerksamkeit auf die Frage nach den Kriterien, die diese Raumkonstrukte charakterisieren. Bisher sind vor allem klassische funktional-qualitative Merkmale erarbeitet worden, die auch im Kontext der nun seit den 80er Jahren laufenden Dikussion um Weltstädte angewandt werden (vgl. Blotevogel 1998a,b). Doch für die Erforschung dieser Räume muss noch eine Ebene systemisch-struktureller Merkmale hinzugefügt werden, durch die der politische und sozioökonomische Entwicklungsprozess in den Vordergrund gelangt und die politikwissenschaftliche und systemtheoretische Analyse von metropolitanen Akteurs- und Handlungssystemen auf der Basis einer Theorie der Subpolitik ermöglicht wird (vgl. BECK 1998).
In der Kombination der funktional-qualitativen und systemisch-strukturellen Merkmale lassen sich Metafunktionen generieren, die zunächst zu einer einfacheren Beschreibung Europäischer Metropolregionen führt.

Die verschiedenen Merkmale einer Metropolregion lassen sich nicht immer ausschliesslich nur einer Metafunktion zuordnen. Beispielsweise wirken Innovationen auch regulierend, denn sie setzen Trends, implementieren neue Standards oder sorgen über Patente für Wettbewerbsvorteile. Als «Gateways» wirken nicht nur physische Infrastrukturen, wie Flughäfen, sondern auch international integrierte Unternehmen, die personifizierbare «Gateways» zu den entsprechenden Märkten bilden können.

\section{Metafunktionen Europäischer Metropolregionen}

- Metafunktion der Technologieentwicklung und Innovation

- Metafunktion des Gateways zwischen metropolitanen, nationalstaatlichen und internationalen Räumen und Märkten

- Metafunktion der politischen und wirtschaftlichen Regulation nationaler und internationaler Politik und Ökonomie, vor allem im Bereich des Finanzsektors 


\subsection{Analyse der Europäischen Metropolregion Zürich}

Die folgende Sammlung von Hinweisen zur Gestalt und Struktur der Europäischen Metropolregion Zürich (EMRZ) versteht sich nicht als vollständige Untersuchung, sondern versucht konzeptionell entlang der drei Metafunktionen Innovation, "Gateway» und Regulation Indizien zu sammeln, inwieweit diese Kriterien von der betrachteten Region bereits «erfüllt» werden. Dies geschieht auf der Datenbasis einer angenommenen Ausdehnung der Metropolregion: der theoretischen Erreichbarkeit vom Flughafen Zürich aus bei einer Autofahrt von 90 Minuten. Hierbei wird man mit dem Dilemma konfrontiert, dass funktionale Metropolregionen eigentlich nicht anhand von politischen Grenzen exakt definierbar sind, da sie aus verschiedenen systemischen Funktionensebenen bestehen, die räumlich unterschiedliche Reichweiten besitzen. Den- noch ist es aufgrund der Auswertung von Daten und der Herstellung funktionaler Bezüge notwendig, einen Datenraum zu definieren. Der dadurch entstandene Analyseraum (vgl. Abbildung 6) bildet daher einen Ausgangspunkt für eine konkretere Erforschung funktionaler Bezüge und damit einer immer exakter werdenden Definition der EMRZ.

\subsection{Innovationsfunktion}

\section{"High-Service»-Standort}

Hochwertige, wissensintensive Dienstleistungen können in Abwandlung des Begriffs «High-Tech» auch als «High-Service» bezeichnet werden. Sie sind hoch innovativ, wenn das auch beim Blick auf «High-Tech» oft in Vergessenheit gerät. Aufgrund ihres Bedarfs an qualifizierten, auch internationalen Arbeitskräften, und der Notwendigkeit der Nähe zu verschiedenen Unternehmen für das Design und die Erstellung komplexer

\begin{tabular}{|c|c|c|c|}
\hline $\begin{array}{l}\text { Wirtschaftszweige } \\
\text { SQ = Standortquotient }\end{array}$ & $\begin{array}{c}\text { Beschäftigte } \\
1985\end{array}$ & $\begin{array}{c}\text { Beschäftigte } \\
1998\end{array}$ & $\begin{array}{l}\text { Veränderung } \\
1985-1998 \text { in \% }\end{array}$ \\
\hline $\begin{array}{l}\text { Kreditgewerbe, Schweiz } \\
\text { - Metropolregion Zürich } \\
\text { - Stadt Zürich } \\
\text { - SQ Stadt Zürich in Metropolregion Zürich } \\
\text { Anteil der Metropolregion an Schweiz } \\
\text { gesamt }\end{array}$ & $\begin{array}{r}108^{\prime} 537 \\
69^{\prime} 528 \\
31^{\prime} 200 \\
3,06 \\
64,1 \%\end{array}$ & $\begin{array}{r}117 ' 238 \\
80 ' 198 \\
38^{\prime} 085 \\
3,67 \\
68,4 \%\end{array}$ & $\begin{array}{r}+8,0 \\
+15,3 \\
+22,1\end{array}$ \\
\hline $\begin{array}{l}\text { Versicherungsgewerbe, Schweiz } \\
\text { - Metropolregion Zürich } \\
\text { - Stadt Zürich } \\
\text { - SQ Stadt Zürich in Metropolregion Zürich } \\
\text { Anteil der Metropolregion an Schweiz } \\
\text { gesamt }\end{array}$ & $\begin{array}{r}51 ' 251 \\
36 ' 873 \\
10^{\prime} 465 \\
1,94 \\
71,9 \%\end{array}$ & $\begin{array}{r}60 ’ 699 \\
45^{\prime} 746 \\
12^{\prime} 447 \\
2,10 \\
75,4 \%\end{array}$ & $\begin{array}{l}+18,4 \\
+24,1 \\
+18,9\end{array}$ \\
\hline $\begin{array}{l}\text { Informatikdienste, Schweiz } \\
\text { - Metropolregion Zürich } \\
\text { - Stadt Zürich } \\
\text { - SQ Stadt Zürich in Metropolregion Zürich } \\
\text { Anteil der Metropolregion an Schweiz } \\
\text { gesamt }\end{array}$ & $\begin{array}{r}15^{\prime}, 872 \\
11^{\prime} 982 \\
3,338 \\
1,90 \\
75,5 \%\end{array}$ & $\begin{array}{r}42 ' 198 \\
33 \cdot 764 \\
5,439 \\
1,25 \\
80,0 \%\end{array}$ & $\begin{array}{l}+165,9 \\
+181,8 \\
+62,9\end{array}$ \\
\hline $\begin{array}{l}\text { Dienstleistungen für Unternehmen, Schweiz } \\
\text { - Metropolregion Zürich } \\
\text { - Stadt Zürich } \\
\text { - SQ Stadt Zürich in Metropolregion Zürich } \\
\text { Anteil der Metropolregion an Schweiz } \\
\text { gesamt }\end{array}$ & $\begin{array}{r}180^{\prime} 911 \\
124 ' 485 \\
32 ' 467 \\
1,78 \\
68,8 \%\end{array}$ & $\begin{array}{r}266^{\prime} 449 \\
185^{\prime} 653 \\
38^{\prime} 043 \\
1,58 \\
69,7 \%\end{array}$ & $\begin{array}{l}+47,3 \\
+50,0 \\
+17,2\end{array}$ \\
\hline
\end{tabular}

Abb. 3: Veränderung der Beschäftigtenzahlen und Standortquotienten von «High-Service»-Branchen in der Metropolregion Zürich

Employee and location site quotient fluctuation of high-service industries in the metropolitan region of Zurich Changement des statistiques relatives aux personnes occupant un emploi et des quotients de localisation dans les branches «high-service» de la région métropolitaine de Zurich

Quelle: Bundesamt Für Statistik, Daten der Arbeitsstättenzählung; eigene Berechnungen 
Lösungen ist auch in der EMRZ ein typischer Metropolregionenprozess ersichtlich. Abbildung 3 zeigt diese Entwicklung für den Zeitraum 1985-1998 anhand der «High-Service»-Branchen Kreditgewerbe, Versicherungsgewerbe, Informatikdienste und Dienstleistungen für Unternehmen. Alle vier haben schweizweit an Beschäftigten zugelegt, die Informatikdienste sogar um $166 \%$. Während der Metropolregionenkern (Stadt Zürich) seine Vormachtstellung als Hauptstandort des Finanzplatzes ausbaut und die Hauptsitze von inund ausländischen Finanzinstitutionen sich auf dichtestem Raum innerhalb der Stadt konzentrieren, ist das Wachstum der weiteren «High-Service»-Branchen vor allem in die Agglomerationsgemeinden um die Stadt Zürich sowie in den weiteren Kernstädten und entlang der Entwicklungsachsen der Metropolregion stärker geworden. Dabei nimmt allerdings die Dichte von Standorten mit zunehmender Distanz von der Stadt Zürich ab.

\section{Produktionsstandort}

Der Strukturwandel Richtung Dienstleistungsgesellschaft hat auch in der Schweiz zu einem Bedeutungsverlust des Produzierenden Gewerbes geführt, was mit einem entsprechenden Abbau von Arbeitsplätzen einherging (vgl. Abbildung 4). Das stark von der Informationsvermittlung abhängige Verlags-, Druckund Vervielfältigungsgewerbe konnte in der EMRZ seinen schweizweiten Beschäftigtenanteil halten, die chemische Industrie, der Maschinenbau und die Branche «Medizinische und Präzisionsinstrumente» bauten nicht nur absolut Beschäftigung ab, sondern verloren besonderes in der Metropolregion und hier vor allem in der Stadt Zürich an Bedeutung. Der stärkere

\begin{tabular}{|c|c|c|c|}
\hline $\begin{array}{l}\text { Wirtschaftszweige } \\
S Q=\text { Standortquotient }\end{array}$ & $\begin{array}{c}\text { Beschäftigte } \\
1985\end{array}$ & $\begin{array}{c}\text { Beschäftigte } \\
1998\end{array}$ & $\begin{array}{l}\text { Veränderung } \\
1985-1998 \text { in \% }\end{array}$ \\
\hline $\begin{array}{l}\text { Verlag, Druck, Vervielfältigung, Schweiz } \\
\text { - Metropolregion Zürich } \\
\text { - Stadt Zürich } \\
\text { - SQ Stadt Zürich in Metropolregion Zürich } \\
\text { Anteil der Metropolregion an Schweiz } \\
\text { gesamt }\end{array}$ & $\begin{array}{r}65^{\prime} 444 \\
51^{\prime} 641 \\
11^{\prime} 132 \\
1,47 \\
78,9 \%\end{array}$ & $\begin{array}{r}55^{\prime} 937 \\
44^{\prime} 180 \\
8,368 \\
1,46 \\
79,0 \%\end{array}$ & $\begin{array}{l}-14,5 \\
-14,4 \\
-24,8\end{array}$ \\
\hline $\begin{array}{l}\text { Chemische Industrie, Schweiz } \\
\text { - Metropolregion Zürich } \\
\text { - Stadt Zürich } \\
\text { - SQ Stadt Zürich in Metropolregion Zürich } \\
\text { Anteil der Metropolregion an Schweiz } \\
\text { gesamt }\end{array}$ & $\begin{array}{r}77^{\prime} 328 \\
58^{\prime} 529 \\
1^{\prime} 650 \\
0,19 \\
79,8 \%\end{array}$ & $\begin{array}{r}61^{\prime} 841 \\
47^{\prime} 064 \\
798 \\
0,13 \\
76,1 \%\end{array}$ & $\begin{array}{l}-15,7 \\
-19,6 \\
-51,6\end{array}$ \\
\hline $\begin{array}{l}\text { Maschinenbau, Schweiz } \\
\text { - Metropolregion Zürich } \\
\text { - Stadt Zürich } \\
\text { - SQ Stadt Zürich in Metropolregion Zürich } \\
\text { Anteil der Metropolregion an Schweiz } \\
\text { gesamt }\end{array}$ & $\begin{array}{r}146^{\prime} 442 \\
124^{\prime} 485 \\
11^{\prime} 634 \\
0,64 \\
85,0 \%\end{array}$ & $\begin{array}{r}109^{\prime} 636 \\
91^{\prime} 253 \\
3{ }^{\prime} 927 \\
0,33 \\
83,2 \%\end{array}$ & $\begin{array}{l}-25,1 \\
-26,7 \\
-66,2\end{array}$ \\
\hline $\begin{array}{l}\text { Medizinische und Präzisionsinstrumente, } \\
\text { Schweiz } \\
\text { - Metropolregion Zürich } \\
\text { - Stadt Zürich } \\
\text { - SQ Stadt Zürich in Metropolregion Zürich } \\
\text { Anteil der Metropolregion an Schweiz } \\
\text { gesamt }\end{array}$ & $\begin{array}{r}69^{\prime} 884 \\
44^{\prime} 340 \\
2{ }^{\prime} 189 \\
0,34 \\
63,4 \%\end{array}$ & $\begin{array}{r}70 ' 165 \\
41^{\prime} 457 \\
1 ' 098 \\
0,20 \\
59,1 \%\end{array}$ & $\begin{array}{l}+0,4 \\
-6,5 \\
-49,8\end{array}$ \\
\hline
\end{tabular}

Abb. 4: Veränderung der Beschäftigtenzahlen und Standortquotienten von Produzierenden Branchen in der Metropolregion Zürich

Employee and location site quotient fluctuation of producing industries in the metropolitan region of Zurich Changement des statistiques relatives aux personnes occupant un emploi et des quotients de localisation dans les branches de production de la région métropolitaine de Zurich

Quelle: BundeSAmT Für Statistik, Daten der Arbeitsstättenzählung; eigene Berechnungen 
Rückgang in der Stadt deutet darauf hin, dass die Funktion als Produktionsstandort von anderen Standorten in der Metropolregion übernommen wird.

Ob innerhalb des betrachteten produzierenden Gewerbes der Forschungs- und Entwicklungs- bzw. Innovationsanteil an Arbeitsplätzen dagegen eine Konzentration erfuhr, lässt sich anhand der Daten derzeit noch nicht feststellen. Der Anteil an Beschäftigten, den die Metropolregion innerhalb der Schweiz in diesen Branchen hat, ist aber trotzdem ähnlich hoch wie in den «High-Service»-Branchen. Dennoch hat die EMRZ in der Branche «Forschung und Entwicklung» zwischen 1985 und 1998 im Vergleich zur Gesamtschweiz deutlich an Bedeutung verloren, obwohl die Zahl der Beschäftigten in diesem Zeitraum anstieg: 1985 arbeiteten 5'811 Beschäftigte in der Forschung und Entwicklung (Anteil an der Schweiz: 73,1\%), 1998 waren es 7'230 (64,0\%). Innerhalb der Metropolregion hat die Stadt Zürich ebenfalls verloren: die Zahl der Beschäftigten sank von 752 auf 653, der Standortquotient, gemessen an der Metropolregion, ging von 0,88 auf 0,70 zurück.

Die Innovationsfunktion der Metropolregion Zürich ist im produzierenden Gewerbe relativ zur ganzen Schweiz erhalten geblieben, im Dienstleistungssektor hat die Innovationsfunktion vor allem durch Konzentration auf «High-Service»-Branchen zugenommen.

\section{3 «Gateway»-Funktion}

Metropolregionen sind allein schon aufgrund ihrer Grösse auch Schnittstellen im Land- und Luftverkehr. Das landseitige "Gateway» der EMRZ, aber auch für die gesamte Schweiz, ist Basel. Weitere grenzüberschreitende Verkehrsströme in grösserem Umfang sind in der Schweiz allenfalls noch in Chiasso auszumachen, wo der Verkehr aber zu einem grossen Teil Tessiner Regionalverkehr mit Italien ist. Der Transitverkehr durch die Schweiz ist gemessen am Gesamtverkehrsaufkommen gering. Die Verkehrsströme in der Schweiz setzen sich aus Binnen- und Pendelverkehr zusammen, grössere internationale Verkehrsströme sind nur in Basel erkennbar. Zürich ist ein nationaler Knoten im Strassenverkehr, der internationale Verkehr ist aber gering, nicht zuletzt bedingt dadurch, dass die einzige internationale Achse (Gotthardachse) die EMRZ nur am Rand durchschneidet.

Auch die internationalen Direktverbindungen mit dem Zug sind relativ schwach. Die Metropolregion Zürich ist zwar in Basel stündlich an das IC-Netz in Deutschland angeschlossen, direkte Verbindungen von der Stadt Zürich zu anderen Europäischen Metropolregionen (EMR) bestehen nur zwei (Paris) bis viermal täglich (Frankfurt, Hamburg, Stuttgart, München). Die einzige mit neun täglichen Direktverbindungen häufiger angebundene EMR ist Mailand. Alle anderen Direktzüge zur EMRZ verkehren nur einmal täglich (z.B. Budapest, Prag, Rom, Barcelona). Zürich wird zwar von einigen Hochgeschwindigkeitszügen angesteuert, die entsprechenden Strecken sind aber weit von Zürich entfernt. Von einer Einbindung in das transeuropäische Hochgeschwindigkeitsnetz kann keine Rede sein.

Aus internationaler Sicht ist daher die EMRZ bezüglich der Verkehrsträger Bahn und Strasse eine Art Loch bzw. eine Randregion in den europäischen Netzen, nicht zuletzt auch, weil die zeitliche Distanz zu anderen internationalen Metropolen sehr gross ist. Für die Einbindung in die internationalen Netzwerke und Märkte gilt: Zürich wird nicht angefahren, Zürich wird angeflogen.

Der Flughafen Zürich gehört nach Verkehrsaufkommen (22,7 Mio. Fluggäste) und dem hohen Anteil an Transitpassagieren (44\%) zu den wichtigen Drehscheiben in Europa. Der Flughafen ist somit das «Gateway» der Schweiz für die internationalen Verbindungen in andere Länder und vom Ausland in die Schweiz. Zürich ist gesamt betrachtet die Schnittstelle nationaler Netze zwischen den Verkehrsnetzen Strasse, Bahn und Flugzeug; als internationaler Verkehrsknoten ist jedoch nur der Flughafen von Bedeutung.

Zur «Gateway»-Funktion gehört auch der Messeund Kongressstandort. Vor allem, um international wettbewerbsfähiger zu werden, schliessen sich die grösseren Messestandorte Basel und Zürich innerhalb der Metropolregion zur Messe Schweiz AG zusammen. Mit über 2 Mio. Besuchern jährlich erreichen sie nach dem Zusammenschluss etwa die Grösse der Messe München. Auch bezüglich Kongresskapazitäten ist Nachholbedarf erkennbar, der in den letzten Jahren auch ins Bewusstsein und Blickfeld gerückt ist. Vor allem der Neubau des international ausgezeichneten Kongresszentrums in Luzern hat den Druck erhöht, auch in Zürich entsprechende moderne Kapazitäten aufzubauen. Ein intraregionaler Wettbewerb könnte hier positive Effekte für die Metropolregion als Ganzes haben.

\subsection{Regulationsfunktion}

\section{Politische Regulationsfunktion}

Bern als Bundeshauptstadt liegt am Rand der EMRZ, ob innerhalb oder schon ausserhalb, ist schwer zu entscheiden. Da hier ein wichtiger Teil der politischen Regulation angesiedelt ist, muss man funktional Bern hinzuzählen. Der ausgeprägte Föderalismus in der Schweiz führt dazu, dass ein grosser Teil der politischen Steuerung in den Kantonshauptorten liegt innerhalb der Metropolregion sind dies die Hauptorte von 19 der 26 Schweizer Kantone und Halbkantone. Wichtig ist neben der offiziellen, unabhängigen Politik das Lobbying von Interessengruppen. Hier dürfte die Lage Berns zwischen Zürich und Genf und zwischen der deutschen und französischen Schweiz für einen gewissen Ausgleich sorgen. Bezüglich des Finanzstandorts haben Genf und Zürich ähnliche Interessen, insbesondere in der Verteidigung des Bankgeheimnisses. 
Läge das politische Zentrum der Schweiz auch in Zürich, würde sich aber ein Ungleichgewicht zugunsten Zürichs kaum vermeiden lassen. Der Kern der EMRZ, die Stadt Zürich, hat somit kaum direkte politische Regulationsfunktion, wirkt aufgrund ihrer Grösse und ihres wirtschaftlichen Einflusses aber entscheidungsvorbereitend. In Genf dagegen führt die Massierung internationaler Verbände und Organisationen zu einer starken internationalen Regulationsfunktion und bietet hier ein Gegengewicht zu Zürich.

\section{Wirtschaftliche Regulationsfunktion}

Die wirtschaftliche Regulationsfunktion einer internationalen Metropole erstreckt sich in erster Linie auf die Bedeutung in der Steuerung internationaler Märkte. Entscheidend ist dafür, dass in der Metropolregion Hauptsitze international tätiger Unternehmen angesiedelt sind. Die in der EMRZ liegenden Städte Zürich, Basel und Zug sind bekannt als Sitz international tätiger Finanzdienstleister, Chemie- und Pharmakonzerne sowie Holdinggesellschaften. Doch auch 47 der 50 nach ihrem Umsatz grössten auslandsorientierten Industrieunternehmen der Schweiz liegen in der EMRZ.

\section{Finanzstandort}

Die Finanzmärkte haben aufgrund ihrer Querschnittwirkung in allen Branchen eine wichtige Regulationsfunktion: sie steuern Börsengänge, Kapitalerhöhungen, die Liquiditätsversorgung, den Handel mit Risiken usw. (vgl. SASSEN 1999). Die EMRZ steuert aber nicht nur den nationalen Markt, sondern zählt zu den wichtigsten Finanzstandorten weltweit. In internationalen
Rankings rangiert Zürich in der «zweiten Reihe» hinter den Top-Standorten London, New York, Frankfurt und Tokyo, zusammen mit Paris, Singapur oder Frankfurt. In der Anlageberatung und Vermögensverwaltung gehört die Schweiz zu den wichtigsten Standorten überhaupt (ReClus 1989, BRUGGer \& KärCHER 1992, KrätKe 1992, Rossi \& Steiger 1995, Zürcher KantoNALBANK 2000).

Wie aus Abbildung 3 ersichtlich wurde, hat die Metropolregion Zürich innerhalb der Schweiz an Bedeutung zunehmen können. Besonders deutlich wird dies in der Veränderung der Bankenumsätze nach Standorten (vgl. Abbildung 5). Der grösste Gewinner nach Wachstum ist der Standort St. Gallen mit dem Sitz der Raiffeisen-Bank, nach Marktanteil bleibt er aber klein. Nach Anteil am Gesamtumsatz der Banken in der Schweiz steht eindeutig die Stadt Zürich an der Spitze, sie konnte $23,7 \%$ an Marktanteil gewinnen, vor allem durch die Fusion der alten UBS mit dem Basler Schweizerischen Bankverein, durch den die Kontrolle über ein grosses Umsatzvolumen nach Zürich abgewandert ist. Genf als zweiter grosser Schweizer Bankstandort ist Sitz sehr vieler (Auslands-)Banken, hat nach Umsatz aber nur einen Anteil von fünf Prozent am Schweizer Markt, weil viele der Banken vergleichsweise klein sind und über keine bzw. nur kleine Filialnetze in der Schweiz verfügen.

Andererseits ist auch anhand der Beschäftigtenstatistik die Stärkung der Finanzplatzfunktion der Metropolregion im Verhältnis zur gesamten Schweiz erkennbar, während innerhalb der Region starke Verschiebungen zu beobachten sind. Die Beschäftigtenzahlen sind in der ganzen Metropolregion im Kredit-

\begin{tabular}{|c|c|c|c|c|c|c|}
\hline \multirow[t]{2}{*}{$\begin{array}{l}\text { Finanz- } \\
\text { standort }\end{array}$} & $\begin{array}{l}\text { Umsatz in } \\
\text { Mio. CHF }\end{array}$ & $\begin{array}{c}\text { Umsatzanteil } \\
\text { an Schweiz } \\
\text { gesamt }\end{array}$ & $\begin{array}{l}\text { Umsatz in } \\
\text { Mio. CHF }\end{array}$ & $\begin{array}{c}\text { Umsatzanteil } \\
\text { an Schweiz } \\
\text { gesamt }\end{array}$ & $\begin{array}{l}\text { Veränderung } \\
\text { Umsatz in \% }\end{array}$ & $\begin{array}{l}\text { Veränderung } \\
\text { Anteil in \%- } \\
\text { Punkte }\end{array}$ \\
\hline & \multicolumn{2}{|c|}{1993} & \multicolumn{2}{|c|}{1999} & \multicolumn{2}{|c|}{$1993-1999$} \\
\hline Zürich & $753^{\prime} 657.5$ & 53.0 & 1’917'690.6 & 76.7 & 154.5 & 23.7 \\
\hline Genf & $76^{\prime} 643.4$ & 5.4 & $125^{\prime} 881.9$ & 5.0 & 64.2 & -0.4 \\
\hline St. Gallen & $28^{\prime} 365.1$ & 2.0 & $89 ’ 650.2$ & 3.6 & 216.1 & 1.6 \\
\hline Lausanne & $34^{\prime} 246.6$ & 2.4 & $36 ’ 396.2$ & 1.5 & 6.3 & -0.9 \\
\hline Bern & $72^{\prime} 114.8$ & 5.1 & $36 ' 142.2$ & 1.4 & -49.9 & -3.7 \\
\hline Lugano & $15^{\prime} 861.3$ & 1.1 & $35^{\prime} 262.3$ & 1.4 & 122.3 & 0.3 \\
\hline Basel & $231 ' 564.6$ & 16.3 & $33 ’ 746.1$ & 1.3 & -85.4 & -15.0 \\
\hline Sonstige & $208 ׳ 438.7$ & 14.7 & $226{ }^{\prime} 854.9$ & 9.1 & 8.8 & -5.6 \\
\hline Gesamt & 1'420'892.0 & 100.0 & 2'501'624.4 & 100.0 & 76.1 & \\
\hline
\end{tabular}

Abb. 5: Veränderung der Umsätze und Umsatzanteile der Banken der wichtigsten Schweizer Finanzstandorte Fluctuation of turnover and turnover ratios of banks in the most important financial locations in Switzerland Changement du chiffre d'affaires et des parts de chiffre d'affaires des banques des principales places financières suisses

Quelle: Handelszeitung 1994, 2000; eigene Berechnungen 
gewerbe von 69'528 (1985) auf 80'198 Beschäftigte (1998) massiv gestiegen. In der Metropolregion hat bei den Konzentrationsprozessen in den 90er Jahren vor allem Basel mit einem Rückgang von 7'550 auf 6'221 Beschäftigte und die Stadt Zug (1985: 1'168 Beschäftigte, Standortquotient: 1,71; 1998: 1'056 Beschäftigte, Standortquotient 1,37) an Beschäftigten im Kreditgewerbe verloren. Ein Wachstum des Finanzplatzes fand vor allem in den Gemeinden des Metropolregionkerns statt (1985 - 1998), d.h. in der Stadt Zürich (+6'885 Beschäftigte) sowie in einigen Gemeinden in der Nähe des Flughafens Zürich, wie beispielsweise Opfikon (+3'924 Beschäftigte), Dübendorf (+735 Beschäftigte), Wallisellen (+323 Beschäftigte).
Der Finanzstandort ist ein typisches Beispiel für ein zentrales Merkmal Europäischer Metropolregionen: ihre verbreitete Polyzentrik. Innerhalb der EMRZ existiert eine räumliche Arbeitsteilung. So sind in Zug Holding- und eine Reihe von Finanzgesellschaften konzentriert, das international führende Clearingund Settlement-Unternehmen Sega-Intersettle hat ihr operatives Zentrum in Olten, während das Management jedoch in Zürich residiert, Basel wiederum ist Sitz der Bank für Internationalen Zahlungsverkehr, einem wichtigen Regulator im internationalen Finanzsystem. St. Gallen als Standort der international anerkannten Universität St. Gallen ist so etwas wie die Kaderschmiede der EMRZ.

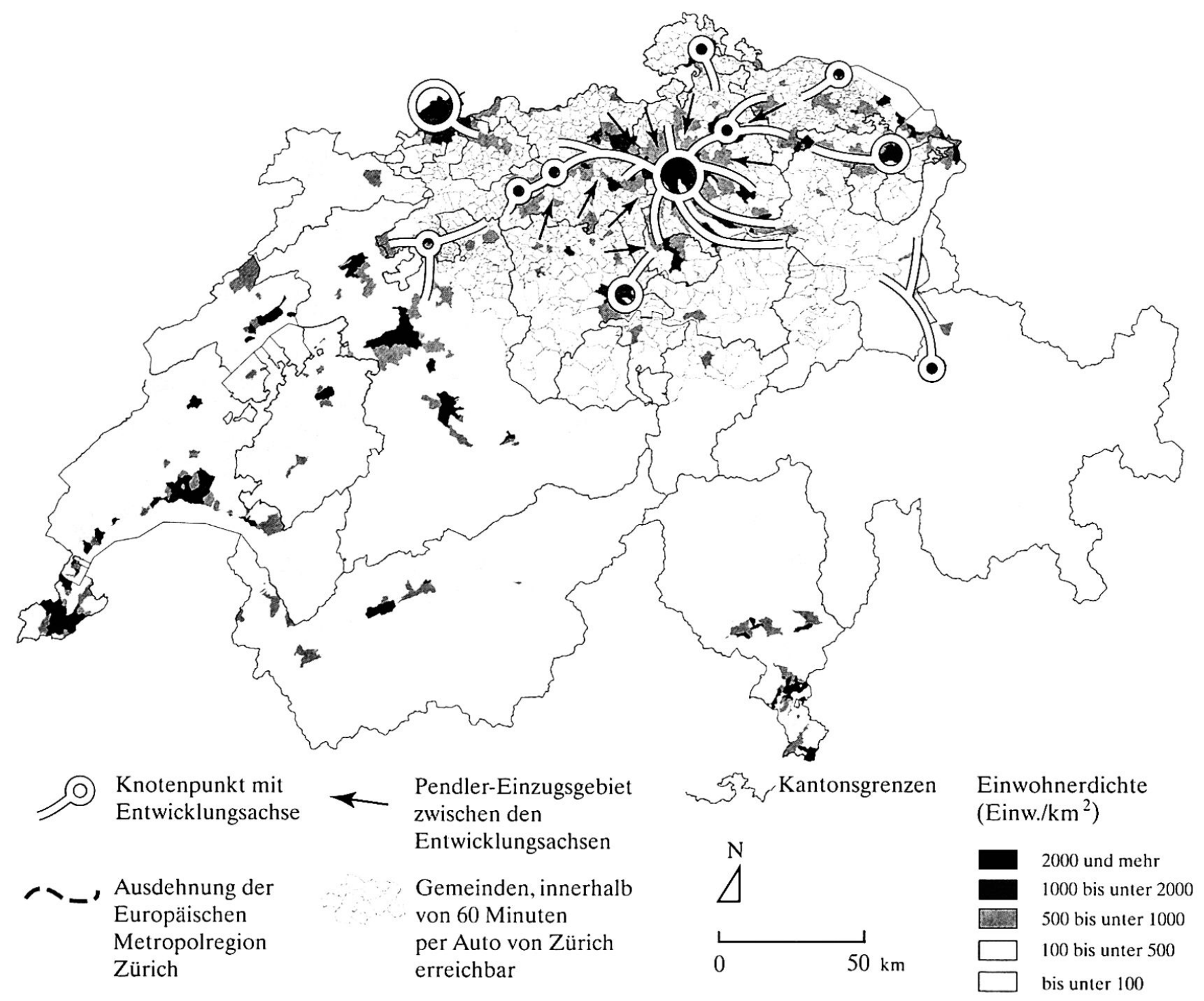

Abb. 6: Entwicklungs- und Verflechtungsachsen in der und um die Europäische Metropolregion Zürich Development corridors and traffic nodes in and around the European metropolitan region of Zurich Axes de développement et de ramification dans et autour de la région métropolitaine européenne de Zurich Quelle: ORL ETHZ; Kartographie: L. GlanZmanN 


\section{Die EMRZ - Verhältnis zwischen dem Metropolen- kern und seiner Basis}

Für die EMRZ ergibt sich eine interessante räumlichfunktionale Struktur. Sie ist einerseits mit jener der Region Frankfurt/Rhein-Main vergleichbar, besitzt andererseits aber einige strukturelle Besonderheiten, die im einzelnen Gegenstand gezielter Forschungen werden müssen, um diese Metropolregion besser räumlich charakterisieren zu können.

Nachdem schon in Punkt 4.1 dargestellt worden ist, wie die EMRZ in diesem Beitrag für eine erste analytische Einordnung räumlich abgegrenzt ist, entsteht folgendes Bild (vgl. Abbildung 6). Der im Verhältnis kleine Metropolregionenkern wird deutlich von der Stadt und der Agglomeration Zürich gebildet. Von hier aus werden die drei Metafunktionen gesteuert. Doch reicht die Grösse der Agglomeration Zürich bei weitem nicht aus, um die Metafunktionen inhaltlich zu erfüllen. Die Region Zürich ist angewiesen auf eine Metropolregionenbasis, die zum einen aus einem polyzentrischen Städtesystem und den dazugehörenden urbanisierten Räumen besteht. Durch sie werden einzelne Funktionen, beispielsweise als Back-OfficeStandorte oder als Standorte von KMU, die innerhalb einer horizontalen Wertschöpfungskette integriert sind, mitgestaltet und damit die Entwicklungsachsen der EMRZ bestimmt. Zum anderen müssen auch ländlich strukturierte Räume als integrativer Bestandteil der Metropolregionen berücksichtigt werden, die beispielsweise durch Erholungs- und Freizeitfunktionen zum Aspekt der Lebensqualität innerhalb der Metropolregion beitragen. Aus dieser Sichtweise ergeben sich interessante Perspektiven für die Schweizerische Raumordnungs- und Regionalpolitik. Zum Beispiel werden Fragen der Siedlungsentwicklung, des Verkehrs oder die Rolle des Flughafens Zürich in ein anderes Licht gestellt, als bei der ausschliesslichen Betrachtung des Kantons beziehungsweise der Agglomeration Zürich.

Das Verhältnis von der Basis zum Metropolenkern kann unterschiedlich stark ausgeprägt sein. Schematisch lassen sich mindestens vier Fälle denken, die das Grössenverhältnis des Metropolenkerns zu seiner Basis beschreiben:

- Die grossen internationalen Metropolen weisen weltweite Konzentrationen von Metropolfunktionen auf, die von einer sehr grossen Basis gestützt werden: New York, Paris, London.

- Der Metropolenkern kann in seinem Umfang zur Basis gross sein, wie beispielsweise in Genf oder Singapur. Oft zeigen sich dann Spezialisierungen auf eine oder zwei Metafunktionen.

- Zwischen den beiden genannten Fällen liegen Metropolregionen, die international kleine, aber national bedeutende «Gateway»- und mindestens politische Regulationsfunktionen besitzen; dies sind vor allem Hauptstädte mittlerer Dimension in kleineren Ländern, wie z.B. Prag, Oslo oder Zagreb.

- Der Metropolenkern kann in seinem Umfang im Verhältnis zur Basis klein sein, wie das bei der europäischen Metropolregion Frankfurt oder beispielsweise auch Zürich der Fall ist.

Unterschiede lassen sich auch in der Stärke der Kopplung zwischen Basis und Metropolenkern finden. Die Metropolfunktionen können recht abgehoben sein (schwache Kopplung), wenn die Metropolfunktionen stärker horizontal integriert sind, wie das bei den internationalen Organisationen in Genf zu beobachten ist. Sie können aber auch eng mit der Basis verzahnt sein, wie das vor allem bei einer grossen Zahl international tätiger Unternehmen in einer Metropolregion der Fall sein dürfte, die neben dem Hauptsitz auch Produktionsstätten in der Metropolregion besitzen (starke Kopplung). Bei starken Kopplungen zwischen den Metropolfunktionen und ihrer Basis dürfte auch das Wachstum beider eng gekoppelt sein. Schwache Verbindungen könnten dagegen zu einer Entkopplung im Wachstum von Metropolenkern und Basis führen. Für die EMRZ tauchen daher einige spezifische Strukturen auf, die im einzelnen noch konkreter analysiert werden müssen. Im Zentrum steht vor allem die Frage nach der funktionalen Anbindung Basels, das durch seine engen Verflechtungen zu den grenznahen Räumen Deutschlands und Frankreichs über eine eigene regionale Struktur verfügt. Inwieweit trägt Basel funktional zur Europäischen Metropolregion Zürich bei? Wie muss die Beziehung der Bundeshauptstadt Bern zur EMRZ charakterisiert werden? Wie muss man mit dem grenznahen süddeutschen Raum umgehen; ist er ein Bestandteil der EMRZ? Und letztlich müsste eine Auseinandersetzung mit der räumlich weiter entfernten Agglomeration Chur erfolgen, da ihr wirtschaftlicher Bezugsraum deutlich die EMRZ ist?

Für die Erforschung dieser Fragestellungen ist es notwendig, sich vom in der Schweiz verbreiteten Agglomerationsdenken zu lösen. Dabei geht es nicht darum, Agglomerationsprobleme nicht mehr zu diskutieren. Agglomerationen funktionieren systemisch anders. Bei der EMRZ handelt es sich um ein europäisches Phänomen. In den Metropolregionen werden die zukünftigen Systemstrukturen demokratisch-föderaler Politik in Europa entwickelt. Diese Situation bereitet kooperativen Zusammenarbeitsformen zwischen Wirtschaft und Politik, die meist auf der Basis von Initiativverbünden metropolitane Politik auf der subpolitischen Ebene übernehmen, den Boden. Auch in der EMRZ wird man sich diesen Prozessen stellen müssen. 


\section{Literatur}

Andersson, A. \& D.E. Andersson (Hrsg.) (2000): Gateways to the Global Economy. - Cheltenham, Northampton: Edward Elgar.

BECK, U. (1986): Risikogesellschaft. Auf dem Weg in eine andere Moderne. - Frankfurt am Main: Suhrkamp.

BECK, U. (1998): Wie wird Demokratie im Zeitalter der Globalisierung möglich? - In: BEcK, U. (Hrsg.) (1998): Politik der Globalisierung: 7-66. - Frankfurt am Main: Suhrkamp.

BonNeviLle, M. (1994): Internationalization of Noncapital Cities in Europe: Aspects, Processes and Prospects. - In: European Planning Studies 2.3: 267-285.

Blotevogel, H.H. (1998a): Metropolen als Motor der Raumentwicklung und als Gegenstand der Raumordnungspolitik. - In: Forschungs- und Sitzungsberichte der Akademie für Raumforschung und Landesplanung 203: 62-70.

Blotevogel, H.H. (1998b): Europäische Metropolregion Rhein-Ruhr. Theoretische, empirische und politische Perspektiven eines neuen raumordnungspolitischen Konzepts, Institut für Landes- und Stadtentwicklungsforschung 135, Dortmund.

BRUGGer, E.A. \& T. KÄrChER (1992): Weltstädte Schweizer Städte. - Bericht 5 des NFP Stadt und Verkehr. - Zürich.

BUNDESMINISTERIUM FÜR RAUMORDNUNG, BAUWESEN UND STÄDTEBAU (2000): Raumordnungsbericht 2000. Berichte 7. - Bonn.

Bundesamt Für Statistik (Hrsg.) (1999): Die Grossregionen der Schweiz. - Neuenburg.

CERny, P.G. (1998): Globalisierung und die neue Logik des kollektiven Handelns. - In: BECK, U. (Hrsg.) (1998): Politik der Globalisierung. - Frankfurt am Main: 263296.

Dangschat, J.S. (2001): Hamburg vor Köln und München, Berlin deutlich dahinter, Leipzig abgeschlagen - Warum und für wen der «Unsinn von Rankings» Sinn macht. - In: Nachrichten der Akademie für Raumforschung und Landesplanung 1/2001: 1-3.

Dear, M.J. (2000): The Postmodern Urban Condition. - Oxford: Malden.

Dielemann, F.M. \& A. Faludi (1998): Randstad, Rhine-Ruhr and Flemish Diamond as One Polynucleated Macro-region? - In: Tijdschrift voor Economische en Sociale Geografie 89.3: 320-327.

EuropäISChe Kommission (Hrsg.) (1999): EUREK Europäisches Raumentwicklungskonzept. - Luxemburg.

European Metropolitan Regions Project (1999): Strategies for Sustainable Development of European Metropolitan Regions. - Evaluation Report. Submitted to the European Regional Conference «European Metropolitan Regions», Essen 15.-17. September 1999. Goodman, J. (1998): Die Europäische Union: Neue Demokratieformen jenseits des Nationalstaats. - In:
Beck, U. (Hrsg.) (1998): Politik der Globalisierung. Frankfurt am Main:331-419.

Habermas, J. (1998): Jenseits des Nationalstaats? Bemerkungen zu Folgeproblemen der wirtschaftlichen Globalisierung. - In: BECK, U. (Hrsg.) (1998): Politik der Globalisierung. - Frankfurt am Main: 67-84.

Handelszertung (1994): Top 2000. Die grössten Unternehmen der Schweiz. - Zürich.

HANDElszeitung (2000):Top 2000. Die grössten Unternehmen der Schweiz. - Zürich.

Hitz, H., Keil, R., Lehrer, U., Ronneberger, K., Schmid, C. \& R. WolfF (Hrsg.) (1995): Capitales Fatales: Urbanisierung und Politik in den Finanzmetropolen Frankfurt und Zürich. - Zürich.

KrätKE, S. (1992): Hierarchie und Vernetzung im Europäischen Städtesystem. - FSP 1-06 Stadtökonomie, Diskussionsbeitrag 13, Technische Universität Hamburg-Harburg, Hamburg.

Kruse, Ch. (1999): Die Subpolitisierung der Raumordnung - Wo die Innovation Druck macht. - In: DISP 4/1999: 26-29.

KruSE, Ch. (2000): «Olten» - Ich sag bloss «I'm near from Zurich» - und alle wissen Bescheid. - In: DISP 3/2000: 44-48.

Marcuse, P. \& R. van Kempen (eds) (2000): Globalizing Cities. A New Spatial Order? - Oxford: Malden.

Michel, D. (1998): Das Netz der europäischen Metropolregionen in Deutschland. - In: Raumforschung und Raumordnung 56.5/6: 362-367.

Moтzкus, A. (2000): Zur Bedeutung der höherwertigen unternehmensorientierten Dienstleistungen für die Entwicklung der Metropolregionen Westdeutschlands. - In: Raumforschung und Raumordnung 58.4: 265-275.

OECD (2000): Cities for Citizens. Improving Metropolitan Governance. Working Party on Territorial Policy in Urban Areas. - Report DT/TDPC/URB (2000)4. Paris.

Reclus (1989): Les Villes Européenes. - Un rapport pour la DATAR. La documentation francaise. - Paris. Ritrer, E.H. (1997): Europäische Metropolregion Rhein-Ruhr oder: Kann aus einer Städte-Agglomerationen eine Metropolregion werden? - In: Forschungsund Sitzungsberichte der Akademie für Raumforschung und Landesplanung 202: 156-170.

Rossi, A. \& C. Steiger 1995: Die Attraktivität des Zürcher Wirtschaftsraumes. - Teilbericht zur Wirtschaftsstudie über den Kanton Zürich, ausgeführt im Auftrag des Zürcher Regierungsrates und der Zürcher Kantonalbank. - Zürich.

SASSEN, S. (1991): The Global City: New York, London, Tokyo. - Princeton.

Sassen, S. (1994): Cities in a World Economy. London.

SASSEn, S. (1999): Global Financial Centers. - In: Foreign Affairs 78: 75-87.

Scotr, A. (Hrsg.) (2001): Global-City-Regions: Trends, Theory, Policy. - Oxford: Oxford University Press. 
Soja, E.W. (2000): Postmetropolis. Critical Studies of Cities and Regions. - Oxford, Malden.

TAYLOR, P.J. \& D.R.F. WALKeR (2001): World Cities: A First Multivariate Analysis of their Service Complexes. - In: Urban Studies 38.1:23-47.

Thierstein, A., Schuler, M. \& D. Wachter (Hrsg.) (2000): Grossregionen. Wunschvorstellung oder Lösungsansatz. - Bern, Stuttgart, Wien.

Urban Studies (2001): Special Issue: Polycentric Urban Regions. Vol. 38.4.

Zürcher Kantonalbank (Hrsg.) (2000): Metropole Zürich. Der Wirtschaftsstandort im Vergleich. - Zürich.

\section{Zusammenfassung: Die Europäische Metropolregion Zürich - die Entstehung des subpolitischen Raumes}

Metropolregionen sind die Basis der Europäischen Integration. Doch im Gegensatz zu den tradierten demokratischen, föderalen Politiksystemen handelt es sich bei Europäischen Metropolregionen um nicht institutionalisierte, subpolitische Akteurs- und Handlungssysteme, die nach anderen Zielen operieren. Da diese Metropolregionen funktional immer stärker zusammenwachsen, allerdings keinerlei administrative Regulation besitzen, entstehen vermehrt metropolitane Initiativverbünde, die Teile regionaler Politik übernehmen ohne demokratisch dafür legitimiert zu sein. Somit sind Metropolregionen Handlungsraum der Subpolitik. Auch in der Schweiz ist am Beispiel der Europäischen Metropolregion Zürich ein solcher Entwicklungsprozess zu beobachten. Durch die Metafunktionen Technologieentwicklung und Innovation, «Gateway»- und Regulationsfunktion bildet Zürich ein «Interface» zwischen nationalen und internationalen Netzwerken. Dies wird auch in der Schweiz zu einem neuen politischen Diskurs über die föderalistischen Strukturen führen.

\section{Summary: The European Metropolitan Region of Zurich - the creation of a sub-political area}

Metropolitan regions are the foundation of European integration. However, unlike established democratic and federal political systems, these regions are noninstitutionalised, sub-political entities that operate similar to «urban regimes». As these regions continuously grow together functionally despite the non-existence of an administrative regulation, the number of metropolitan initiator networks are increasing. These are taking over partial aspects of regional politics without being in a politically legitimate position to do so. Consequently, metropolitan regions are the field of activity of sub-politics. In Switzerland, the metropolitan region of Zurich exemplifies such a path of develop- ment. With its meta-functions of technological development and innovation, as well as «gateway» and regulatory functions, Zurich has positioned itself at the interface between national and international networks. This development will lead in Switzerland to a new political discourse about current federal structures.

\section{Résumé: La région métropolitaine européenne de Zurich - L'émergence de l'espace subpolitique}

Les régions métropolitaines forment la base de l'intégration européenne. Cependant, par contraste avec les systèmes politiques démocratiques et fédéraux confirmés, il s'agit, en ce qui concerne les régions métropolitaines européennes, de systèmes subpolitiques d'acteurs et d'action non institutionnalisés, qui s'orientent vers d'autres finalités. Comme ces régions métropolitaines fusionnent toujours davantage fonctionnellement, sans toutefois bénéficier d'une quelconque régulation administrative, apparaissent de manière croissante des groupements d'initiatives métropolitaines qui prennent en charge des parts de politique régionale sans avoir été pour autant légitimés démocratiquement. C'est ainsi que les régions métropolitaines constituent un espace d'action subpolitique. Un tel processus de développement est aussi observable en Suisse à partir de l'exemple de la région métropolitaine européenne de Zurich. Par ses métafonctions formées par le développement technologique et l'innovation d'une part, la fonction de "gateway» et de régulation d'autre part, Zurich constitue une interface entre les réseaux nationaux et internationaux. Cette tendance va aussi conduire en Suisse vers un nouveau discours politique sur les structures fédérales.

Dr. Heiko Behrendt, Dipl. Geogr. Christian Kruse, Institut für Orts-, Regional und Landesplanung (ORL), Fachbereich Raumordnung, Eidgenössische Technische Hochschule Zürich, ETH-Zürich (Hoenggerberg), $\mathrm{CH}-8093$ Zürich.

e-mail:

Behrendt@orl.arch.ethz.ch

Kruse@orl.arch.ethz.ch

Manuskripteingang/received/rentrée du manuscrit: 3.7.2001

Annahme zum Druck/accepted for publication/accepté pour l'impression: 31.10.2001 